\title{
A RESTful Web Service for Information Platform of Intelligent Transportation
}

\author{
Ming Wu \\ School of Electronic and Information Engineering \\ Tongji University \\ Shanghai, China \\ iamwuming@163.com
}

\author{
Min Liu, Jianfeng Lu \\ School of Electronic and Information Engineering \\ Tongji University \\ Shanghai, China \\ lmin@tongji.edu.cn, lujianfeng@tongji.edu.cn
}

\begin{abstract}
With the development of intelligent transportation, a large amount of heterogeneous data has emerged from various sensors and terminal applications. An efficient approach to process data is needed in information platform of intelligent transportation system (ITS). This paper presents a lightweight Representational State Transfer (REST) Web Service to process data and provide service support for up layer application, using JavaScript Object Notation (JSON) and Extensible Markup Language (XML) to exchange data. It focuses on two specific aspects in information platform of ITS: sensor data gathering and emergency notification. The application in ubiquitous network of intelligent transportation project proves its validity and feasibility, and some improvement measures are proposed at the end of the paper.
\end{abstract}

Keywords-RESTful; intelligent transportation; Web Services; data processing;

\section{INTRODUCTION}

Intelligent transport systems (ITS) are advanced applications, which aim to provide innovative services relating to different modes of transport and traffic management and enable various users to be better informed and make safer, more coordinated, and smarter use of transport networks [1].

Sensor web [2], as an important part of ITS, has been largely influenced by the concept of the Internet of Things. It is considered as an infrastructure that enables to collect, model, store, retrieve, share, manipulate, analyze and visualize sensor data/metadata via the World Wide Web (WWW) in a standardized way [3].Along with the widely used of various sensors, people can gather more and more real-time information of the car, road, and parking lot, and so on. With these data, people can be clear of the state of the car, traffic situation, and some other useful information. And the emergency situation can be predicted by analyzing these data. For example, the message of traffic jam can be sent to drivers who are heading that area. People can send message to other cars and relevant departments, making travel more safe and convenient. The development of mobile internet also have a great effect on ITS, many terminate application have been come out, making traveling convenient and smart.

Emergency services is another important part of ITS, which will evolve with the blending of vehicular communication networks with road transportation [4]. This service can notify user the emergency message in time and also can post emergency information to the relevant department.

So it is necessary to build an efficient information platform to process data, serving as a bridge of data share and exchange among different application systems and data sources. In 2001, the Ministry of Science and technology of China appointed ten cities as ITS demonstration cities, including Beijing, Shanghai, Guangzhou, etc. The integrated information platform is an important part in the ITS planning of each city. Meanwhile many scholars and some companies have begun the research and development of the integrated information platform and achieved some valuable results. But this technology is far from mature.

A challenge in building a good information platform of ITS is how to process the large amount of heterogeneous data produced by different kinds of sensors and applications. And another challenge is how to provide general service using these data for up layer applications installed in intelligent terminal. A RESTful Web Service for information system of ITS addressing these problems is designed in this paper. It provides an efficient and flexible service for information platform of ITS. This web service for information system of ITS is to focus on two specific aspects: sensor data gathering and emergency notification.

\section{RELATED WORK}

To design this web service, several efforts have been invested. There are many web service technologies nowadays. Every technology has its own advantage. But for the ITS, RESTful architecture is most suitable [5]. This approach is very simple to understand and can be executed on really any client or server that has HTTP/HTTPS support, which is a good feature for ITS containing so many different devices.

As we know, many diverse sensors are used in the ITS, and they are the part of infrastructure of the ITS. In this web service, XML technology is used to describe the sensor and data model [6]. The well-structured, platform and application independent, self-descriptive, simplicity and many other characteristics made it possible to be independent of any application system and platforms. But XML is not well suited to data-interchange. It carries a lot of baggage, and it doesn't match the data model of most programming 
languages. So in this paper JSON is used to interchange data. JSON [7] is a lightweight data exchange format. Relative to the XML, it is easy for machines to parse and generate. JSON uses a text format that is completely independent of the language, which has obviously higher parsing efficiency and the advantages of easy preparation.

This web service is based on Java EE platform [8], using RESTful Web service frameworks [9, 10], mainly focusing on two aspects, sensor data gathering and emergency notification.

\section{SERVICE ARCHITECTURE}

\section{A. RESTful Service Architecture}

REST is an architectural model for how distributed applications are built, which was originally introduced as an architectural style for building large-scale distributed hypermedia systems [11]. Systems built around the REST architecture are said to be RESTful. Here introduces three import concepts of REST:

- Representation: Data or resources are encoded as representation of the data or resource. These representations are transfer between clients and servers

- $\quad$ State: All of the necessary state needed to complete a request must be provided with the request. The clients and servers are inherently stateless. A client cannot rely on any state to be stored in the server, and the server cannot rely on any state stored in the client. This does not, however, pertain to the data stored by servers or clients, only to the connection state needed to complete transactions.

- Transfer: The representations and the state can be transferred between client and servers. REST is an architectural model that can be efficiently implemented as a combination of the Hypertext Transfer Protocol (HTTP) and TCP/IP. With this instantiation of REST, HTTP requests are used to transfer representations of resources between clients and servers. Uniform Resource Identifiers (URIs) are used to encode transaction states.

RESTful Web services are perceived to be simple because REST leverages existing well-known W3C/IETF standards (HTTP, XML, URI, and MIME) and the necessary infrastructure has already become pervasive. HTTP clients and servers are available for all major programming languages and operating system/hardware platforms, and the default HTTP port 80 is commonly left open by default in most firewall configurations.

The Web Service framework designed in this paper can be demonstrated in Fig.1. This web service uses Java EE platform and MySQL database, which are both open source software. And the smart phone can be an iPhone or an Android phone. Through the web service interface, any application can be design to meet the certain need with the data provided by the web server. And in the future, the vehicle will be smarter and smarter, and more and more applications will be integrated into the intelligent vehicle system.

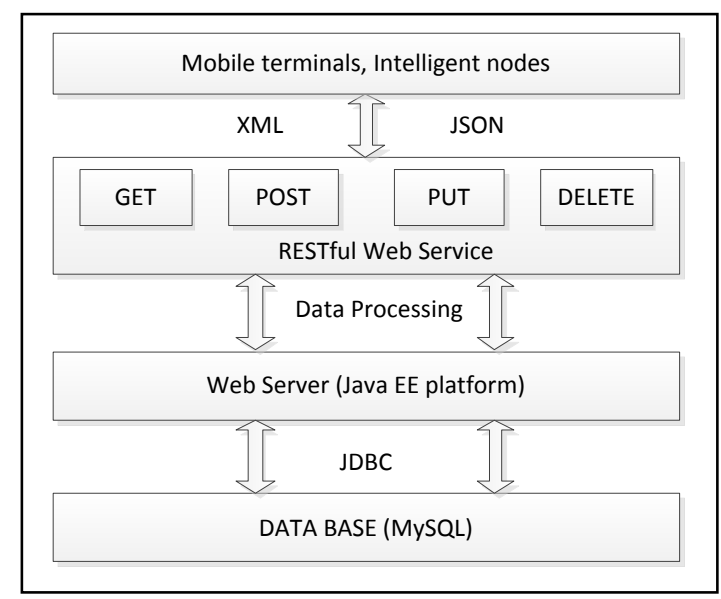

Figure 1. RESTful Web Service architecture

\section{B. Data Processing Architecture}

In the information platform for ITS, there are a lot of sensors data need to be processed efficiently. And at the same time, this platform should provide support for up layer application. This paper proposes a hierarchical architecture system platform for sensor data gathering. And the overview architecture is shown in Fig. 2.

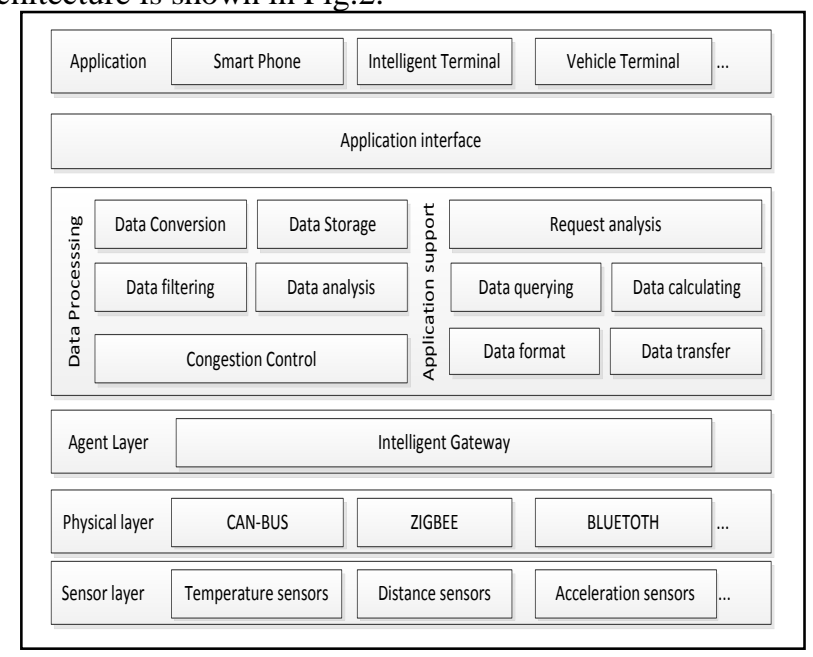

Figure 2. Data processing architecture

Sensor Layer consists of various kinds of sensors installed in the vehicle and route, which is at the bottom logic structure of the ITS system. The original data collection is realized in this layer.

Physical layer is made up of data transmission devices using different kinds of physical transmission methods and protocols (such as ZigBee, CAN-bus), due to different application environment and different needs. It is in charge of communication between the device layer and its upper layers, which regarded as a data transport layer.

Agent layer can be regarded as a transforming layer between physical layer and its upper layers. Physical 
communication protocol used by various type of device is transformed to a certain physical protocol by the intelligent gateway. The function of this layer is protocol conversion.

Data processing layer mainly deals with the massive data generated by the vehicle terminal. Its task includes congestion control of the network, and filtration, storage and analysis of the data. The other function of this layer is providing support for up layer application. When an application send a request, it should give back a right response, including data querying, data analyzing, etc.

This is a RESTful style web service, using REST API to support for the application built in different operation systems platforms, such as Android, Linux, vehicle embedded systems, etc. And via these REST interface, some web pages can be designed easily to show the information that we need in a certain form. For example, historical trajectory of the vehicle at a specific time ranges can be shown by organizing the location data.

\section{FUNCTION REALIZATION}

The implementation of this web service is based on Java EE platform, with the use of SSH and Restlet framework. And it is a platform-independent Web services, with highly versatile. This web service for intelligent transportation mainly focuses on three aspects:

\section{A. Sensor Data gathering}

In the ITS, a lot of sensors are used in the vehicles and routes, etc. And the data collected by these sensors are heterogeneous, massive and uncertain. To gather the useful data efficiently, the following steps are taken:

\section{1) Sensor model defination}

In this step, a set of standards describing sensor metadata and values need to be made. XML technology is adopted to encode the sensor data model, which is a well-structured, platform and application independent, self-descriptive language. Because JSON is more suitable for light-weight RESTful web service, the XML data will be converted to JSON in the transmission.

\section{2) Data congesture control}

The origin sensor data is transfer to intelligent gateway via some short distance communication technology, such as ZigBee, CAN-bus. Then the data was transport to web server through TCP/IP protocol.

Then here comes an important question, congestion control. Because massive data is produced form all kinds of sensors, it is easy to cause congestion and lead to vicious cycle when the bandwidth through gateway is not enough. So Random Early Detection (RED) [12] is adopted to control congestion. This algorithm will monitor the length of the data packet queuing in router. Before the cache is filled, the data packets will be discarded at a certain probability once found congestion approaches. So the congestion can be controlled from the source. The RED algorithm consists of two parts, one is to monitor the average queue length and the other is to judge whether the data packet should be discarded.

The RED algorithm can keep the average queue length at a low level by early discard packets randomly and it is useful for the absorption of short-term burst data stream. What is more, due to the use of RED algorithm, we can avoid the global synchronization problem of removal tail algorithm.

3) Data process

Data processing contains data filter, data store, and data analysis.

The data collected from sensors contains a lot of duplicate data, and not all the data are useful. Only valuable data need to be saved in data base.

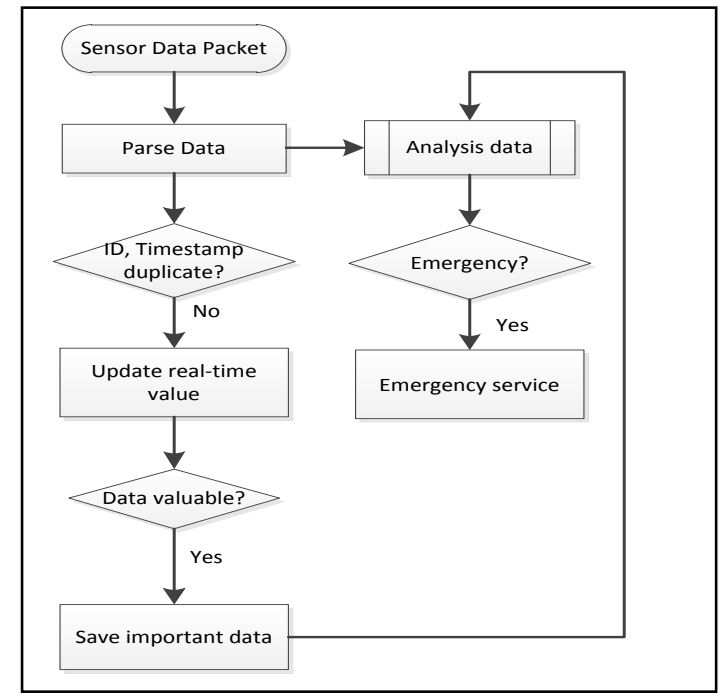

Figure 3. Data processing procedure

\section{B. Emergency Notification}

Since vehicles and other transport infrastructures have sent their data to the web server through wireless network, many emergency services can be developed.

Emergency service in ITS contains many aspects, such as route state, vehicle condition, traffic accidents, etc. Through this service, the vehicle to vehicle (V2V) and vehicle to infrastructure (V2I) system can be easily designed. The emergency messages can be push to users and transport department automatically in time so that a lot of accidents or drivers' wrong decision can be prevented.

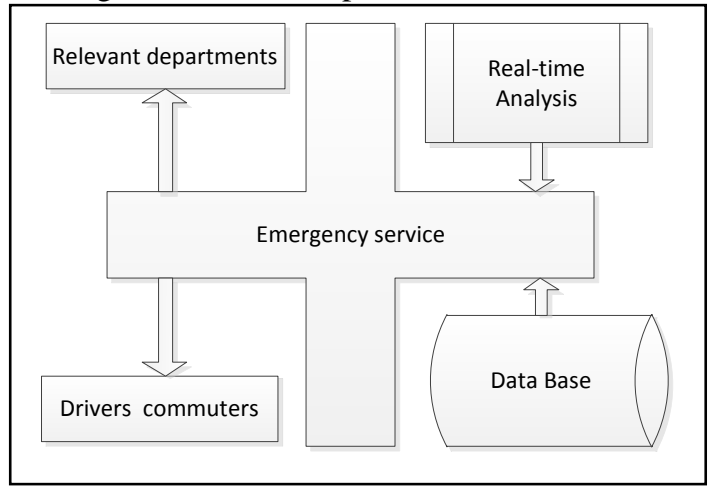

Figure 4. Emergency notificaiton service 
The emergency service also uses RESTful interface, any clients having HTTP/HTTPS support can use this service, which is really convenient.

Take the route state notification for example. By analyzing the data collected, the average velocity of the vehicles at the same area can be figure out. Then it's easy to know whether the traffic jam happens or not. And the message can be posted to drivers who are heading that area so that the drivers can change their path.

\section{A CASE STUdY}

This web service was used in the intelligent transport ubiquitous network project, which is a Major National Science and technology project.

In this project, several important sensors were integrated into the vehicles, such as temperature sensor, acceleration sensor, distance sensor, etc. The data were transmitted to the server via the $3 \mathrm{G}$ telecommunication networks. As for the parking lots, route, or other transport infrastructure, data were transmitted through $\mathrm{WiFi}$. The web service was developed with Java EE platform, using SSH and Restlet framework. With the web service interface, various applications were designed to meet the specific need.

With this web service, people can check the real-time information of the certain route and parking lot, which is very helpful for uses to make a right decision. Many important data about the vehicle were saved in the database, and they can be very useful to predict the failure of the vehicle in the future. What's more, the emergency service can help to prevent accidents, making travel safer. In this project, the emergency service contains traffic jam alarm, automatically emergency call, and relevant department realtime notice, etc.

All the functions of this project have been tested, which proves the feasibility of this web service.

\section{CONCLUSIONS}

This paper designs a lightweight RESTful Web Service to process data and provide service support for up layer application, focusing on massive heterogeneous data processing and emergency notification. And it performed quiet well in the Intelligent Transport Project, which prove that it can be a good solution for information platform implementation for ITS, serving as a bridge of data share and exchange among different application systems and data sources.

There are also some deficiencies. The sensor deployment is a little tedious and the data process algorithm still needs to be improved, which are the major work in the future research.

\section{ACKNOWLEDGMENT}

Some of research work in this paper is supported by Major National S\&T Projects (No.2011ZX03005-004-01).

\section{REFERENCES}

[1] Gholamreza Khorasani, Ashkan Tatari, Ali Yadollahi and Milad Rahimi, "Evaluation of Intelligent Transport System in Road Safety," in IJCEBS, vol. A247, Volume 1, Issue 1.2013.

[2] Yudong Tian; Geiger, J.V.; Hongbo Su; Kumar, S.V.; Houser, P.R., "Middleware-Based Sensor Web Integration," Selected Topics in Applied Earth Observations and Remote Sensing, IEEE Journal of , vol.3, no.4, pp.467,472, Dec. 2010

[3] Mohsen Rouached, Sana Baccar, Mohamed Abid, "RESTful SensorWeb Enablement Services forWireless Sensor Networks" in Services (SERVICES), pp.65 - 72, IEEE Eighth World Congress 2012.

[4] Martinez, F.J.; Chai-Keong Toh; Cano, J.-C.; Calafate, C.T.; Manzoni, P., "Emergency Services in Future Intelligent Transportation Systems Based on Vehicular Communication Networks," Intelligent Transportation Systems Magazine, IEEE , vol.2, no.2, pp.6,20, Summer 2010

[5] Cesare Pautasso, Olaf Zimmermann, and Frank Leymann. "Restful web services vs. "big" web services: making the right architectural decision", in Proceeding of the 17th international conference on World Wide Web, WWW '08, pages 805-814, New York, NY, USA, 2008. ACM

[6] Jing Zhang; Bo Lang; Yawei Duan, "An XML Data Placement Strategy for Distributed XML Storage and Parallel Query," Parallel and Distributed Computing, Applications and Technologies (PDCAT), 2011 12th International Conference on , vol., no., pp.433,439, 20-22 Oct. 2011

[7] Guanhua Wang ,"Improving Data Transmission in Web Applications via the Translation between XML and JSON" ,in Communications and Mobile Computing (CMC), 2011, pp.182-185.

[8] Shuang Liu; Peng Chen, "Developing Java EE Applications Based on Utilizing Design Patterns," Information Engineering, 2009. ICIE '09. WASE International Conference on , vol.2, no., pp.398,401, 10-11 July 2009

[9] Shuang Liu; Peng Chen, "Developing Java EE Applications Based on Utilizing Design Patterns," Information Engineering, 2009. ICIE '09. WASE International Conference on , vol.2, no., pp.398,401, 10-11 July 2009

[10] Hongjun Li, "RESTful Web service frameworks in Java," Signal Processing, Communications and Computing (ICSPCC), 2011 IEEE International Conference on , vol., no., pp.1,4, 14-16 Sept. 2011

[11] Cesare Pautasso and Erik Wilde. "Restful web services:principles, patterns, emerging technologies" in Proceedings of the 19th international conference on World wide web, WWW' 10, pp. 13591360, New York, NY, USA, 2010. ACM..

[12] Guan-Yi Su; Ho, C.C. "Random Early Detection Improved by Progressive Adjustment Method " in telecommunication Technologies 2008 and 2008 2nd Malaysia Conference on Photonics, pp. 250 - 253, NCTT-MCP 2008. 6th National Conference 